Reprod. Nutr. Dévelop., 1988, 28 (6 B), 1555-1568.

\title{
Les avortements spontanés à répétition
}

\author{
J. SALAT-BAROUX
}

Service de Gynécologie Obstétrique et Biologie de la Reproduction. Hôpital Tenon, 4, rue de la Chine, 75020 Paris.

\section{Summary. Recurrent spontaneous abortion.}

The process of fertilization in humans, is remarkably inefficent. Spontaneous abortion is estimated to be between 15 and $20 \%$ of all clinical pregnancies, and the early spontaneous abortion rate is closer to $30-50 \%$ of fertilized ova. Not all authors agree on the definition of « recurrent spontaneous abortion » (RSA), so the frequency of repeated pregnancy wastage is difficult to determine; from empirically derived data, it has been estimated to range between 0.4 and $0.8 \%$.

Because of the various etiologies of RSA, their association in determining an abortive event, it is difficult to evaluate their exact incidence. Moreover, their is no prospective study on this subject, so it is advisable to distinguish between the admitted causes, the likely factors, and the etiologies to be evaluated. In the first group, the congenital or acquired müllerian anomalies (especially the septate uterus), represent about $25 \%$ of the RSA, but a lot of problems concerning the physiopathology are still debated, even if the rate of pregnancies after surgery ranges around $50 \%$ in certain series. On the other hand, the genetic factors, identified especially with the banding technique, are undeniable : however, although the rate of chromosomal aberrations in the offspring (Monosomy $X$, Trisony 16 , Triploidy) is very high (50 to $60 \%$ of spontaneous abortions in the first trimester of pregnancy), when couples with usual abortions are subjected to karyotypic analysis, genetic anomalies (especially translocations) are been noted in only $6.2 \%$ of the women and $2.6 \%$ of the men.

In the second group, the infective factors (chlamydiae, toxoplasma and mycoplasma) are difficult to analyse since the serology is not sufficent without a real proof of an endometrial colonization. Among the endocrinological causes, the classical luteal phase deficiency remains a subject of controversy (estimated between 3 and $30 \%$ ) not only for the establishment of the diagnosis, but also for the efficiency of progesterone supplementation. In the third group, the autoimmune diseases, especially systemic lupus erythematosus (SLE) are most likely a cause of fetal wastage, by secreting antibodies that cross-react with the antigenic determinants of the trophoblast. These patients should be screened for lupus like anticoagulant; the therapy with glucocorticoids during pregnancy is still debated. More recently, the immunologic factors have been estimated to play an important role in the etiology of usual abortion : in these cases women share HLA antigens with their partners more frequently than expected. Thus, the presence of paternal antileukocytes antibodies, provided by leucocyte transfusions, semms to be associated with reproductive success and strengthens this hypothesis.

But the "saga " of recurrent spontaneous abortion, is not finished, if we consider recent research on the first stages of embryo development by ultrasound and doppler, biochemical and immunological techniques. 
Invariablement, tous les 10 ans, les communautés scientifiques composées de cliniciens et fondamentalistes sont obligées d'actualiser la question des avortements à répétition. Les résultats sont immanquablement les mêmes : on brûle ce que l'on adorait (l'œuf au bismuth, l'œuf aux antibiotiques, aux hormones et maintenant aux corticoïdes) en raison d'une méthodologie inadéquate et de perspectives toujours renouvelées par les fondamentalistes. Pour clarifier le débat, il faudrait préciser la complexité du sujet, analyser de façon critique les étiologies en retenant surtout celles qui font l'unanimité et enfin définir une attitude prospective à la fois diagnostique et thérapeutique.

\section{1) La première difficulté tient à la définition même des avortements} spontanés à répétition et à leur fréquence. S'il n'y a pas un intérêt primordial à distinguer les femmes qui ont eu deux avortements de celles qui en ont eu trois ou plus, en fait les arguments de discussion deviennent plus nombreux quand on considère les patientes n'ayant eu que des avortements à répétitions et celles qui ont une parité émaillée de naissances vivantes. Bien plus, doit-on conserver une frontière étanche entre les échecs de la reproduction survenus avant la $20^{\mathrm{e}}$ semaine, et dénommés communément avortements, et ceux qui sont postérieurs, se traduisant par des morts in utero, des morts péri- ou néonatales, ou encore des enfants mal formés? La grande majorité des publications consultées, ne tient pas compte de ces distinctions pourtant fondamentales (Rock et Zacur, 1983; Candiani et Fedele, 1987) si l'on considère certaines étiologies en particulier auto-immunes.

Une très grande confusion règne dans l'appréciation de la fréquence de cette pathologie. Comment évaluer le risque d'avortement spontané à répétition, si déjà l'épidémiologie des avortements simple est contestée? Ceux-ci représenteraient 15 à $20 \%$ des grossesses reconnues cliniquement. Or l'on sait que la moitié, sinon $60 \%$ des conceptions humaines (Boué et Boué, 1977) aboutissent à un échec dans les premières semaines de la grossesse. Il serait pourtant très utile de pouvoir évaluer ce taux de "mortalité intra-utérine" surtout si l'on soupçonne une étiologie génétique. Ainsi Leridon (1977) combinant les résultats de Hertig et Rock (1959) d'une part, ceux de French et Bierman (1962) d'autre part, dresse une table complète de mortalité intra-utérine pour 100 ovules exposés au risque de fécondation : le taux d'échec total est de $69 \%$ et de $63 \%$ si l'on s'en tient aux seules fécondations effectives. La sécrétion d'hCG par l'embryon est très précoce dès le $6^{\mathrm{e}}$ jour ; l'étude de l'ascension transitoire de B hCG plasmatiques dans la phase lutéale par plusieurs équipes, ou la prise en compte d'une diminution des taux de plaquettes maternelles par un facteur embryonnaire, confirment les récents résultats rapportés par les équipes de fécondation in vitro: une perte embryonnaire d'environ $50 \%$. La question serait de savoir si ce taux est encore plus important chez les femmes ayant des avortements à répétition. Cependant, si l'on s'en tient aux avortements spontanés cliniquement et échographiquement prouvés, l'estimation générale, mais forcément entachée d'erreurs serait de 0,4 à $0,8 \%$ (Candiani et Fedele, 1987). Mais en fait, la plupart des études épidémiologiques vraiment documentées concluent que cette fréquence est impossible à évaluer dans une population donnée. Par contre le risque de voir survenir un 
avortement spontané à répétition, a été chez la même femme évalué à 22,38 et $73 \%$ respectivement après 1, 2 ou 3 avortements antérieurs (Leridon, 1973). Mais ces chiffres ont été diversement appréciés par d'autres équipes suivant qu'ils font appel à une évaluation mathématique du risque ou à une simple étude rétrospective. Un fait est certain, c'est qu'il s'accroît avec l'âge maternel et le passé gynéco-obstétrical de la patiente.

II) Compte tenu de ces ambiguités de départ, que penser des étiologies, communément admises? Si l'on veut faire une analyse critique des travaux antérieurs, on est bien obligé de constater qu'un certain nombre de points n'ont jamais encore reçu de réponse: 1) dans toutes les statistiques 40 à $50 \%$ des avortements à répétition ne trouvent pas d'étiologie même immunologique. 2) Aucune étude jusque-là n'a été prospective. 3) Dans aucune série il n'est tenu compte du facteur âge dont on sait qu'il augmente indiscutablement la composante génétique défavorable. C'est ainsi qu'on reconnaît aux fibromes utérins un effet abortif dans $15 \%$ des cas d'avortement à répétition, or ces anomalies utérines intéressent les femmes de plus de 35 ans et aucune vérification du caryotype ne permet d'éliminer une étiologie génétique associée ou prédominante. 4) L'épreuve thérapeutique elle-même demande à être analysée, en l'absence très souvent de cas contrôlés et de séries randomisées. Nous y reviendrons.

Si l'on s'en tient aux différentes études rétrospectives publiées (Beer, 1986 ; Rock et Schlaff, 1985), on peut retrouver un certain consensus sur les causes génétiques dont la fréquence est estimée autour de $25 \%$ des cas, les causes utérines congénitales ou acquises de 15 à $20 \%$, les causes endocriniennes autour de $15 \%$, infectieuses de $10 \%$, toutes les autres (métaboliques, toxiques, immunologiques et psychologiques) étant diversement appréciées suivant les centres ou les années de publications. Il apparaît donc logique d'abandonner l'idée de classer ces étiologiques suivant leur ordre de fréquence, mais plutôt de les regrouper en causes certaines et prouvées, causes probables et causes en voie d'évaluation.

A) Dans les causes certaines, on peut regrouper les anomalies utérines et les aberrations chromosomiques.

\section{a) Les anomalies utérines.}

L'incidence des anomalies congénitales dans la survenue des avortements à répétition est diversement appréciée : de 23 à $44 \%$ (Lecontour et al., 1986). De plus le type anatomique de la malformation intervient dans la fréquence de ces accidents : $28 \%$ dans les utérus bicornes bicervicaux, $34 \%$ dans les bicornes unicervicaux, $45 \%$ dans les unicornes et surtout les utérus cloisonnés. Si ces chiffres dans leur ensemble sont grossièrement acceptés, il n'en demeure pas moins un certain nombre de points obscurs: 1) la fréquence exacte de ces anomalies utérines dans la population générale est méconnue, au maximum de 0,1 à $0,5 \% ; 2$ ) de plus leur diagnostic n'est fait avant l'accident que dans 30 à 
$65 \%$ des cas. Il existe donc un biais évident dans l'appréciation de ces chiffres : on ne possède pas de série contrôle puisqu'on ignore le nombre de grossesses totales correspondant aux utérus mal formés; on ne recense que les utérus malformés ayant conduit à des avortements à répétition. Deux questions se posent du même coup : pour quelles raisons ces malformations sont-elles cause d'avortement? Enfin quel est l'effet de la thérapeutique chirurgicale? A la première question, pourtant passionnante sur le plan physiopathologique, il n'y a pas encore de réponse précise : a) L'étroitesse utérine (et le manque d'étoffe) aurait été une bonne raison, si elle n'était contredite par le fait que la répétition des grossesses n'est pas toujours couronnée de succès, et que les avortements pour plus de $50 \%$ d'entre eux surviennent dans le $1^{\text {er }}$ trimestre. $b$ ) Si la béance de l'isthme garde encore de farouches défenseurs, aucune étude prospective et randomisée ne permet de justifier cette conception mécaniste, surtout pour les avortements précoces. $c$ ) La mauvaise décidualisation de l'endomètre, longtemps évoquée, n'a pas résisté à des études histologiques systématiques. d) Mais l'hypothèse d'une vascularisation inadéquate au niveau du site d'implantation demandera à être vérifiée par des études en écho Doppler. En attendant, il semblerait que le type de malformations interviendrait dans le pronostic : c'est ainsi que les utérus cloisonnés seraient les grands pourvoyeurs d'avortement à répétition. Mais ne doit-on pas là aussi être circonspect et considérer que ce type de malformation est la moins fréquente et la plus aisément réparée. Il n'y a, à notre connaissance, aucune étude publiée sur les sites d'implantation de l'œuf, précédant l'avortement, repéré par échographie dans le cadre de ces utérus cloisonnés. Bien plus, il n'y a aucune étude comparée de biopsies d'endomètre suivant le site de prélèvement (cloison ou corps utérin), ce qui est actuellement tout à fait réalisable sous hystéroscopie.

La réponse à la deuxième question pourrait représenter un encouragement : si la série du John Hopkins Hospital (Rock et Schlaff, 1985) est un hymne à la gloire de la métroplastie, si les récentes résections endoscopiques soulèvent un vent d'espoir, d'autres auteurs restent plus circonspects concernant le nombre d'enfants vivants obtenus (Audebert et al., 1983) et soulignent le rôle des traitements médicaux adjuvants en post-opératoire. Mais à la question de savoir s'il faut attendre deux ou trois accidents avant d'intervenir, les réponses sont nuancées: certains (Rudigoz et al., 1985) sont d'emblée interventionnistes, arguant du fait que la répétition des grossesses n'améliorent en rien le pronostic, mais les chiffres qu'ils rapportent ne sont guère significatifs sur le plan statistique. Il faut rapprocher des anomalies congénitales, les troubles du développement utérin chez des patientes exposées à l'administration du Diéthylstilbestrol entre 1949 et 1970. Le nombre d'anomalies peut atteindre $86 \%$ des cas et le taux d'avortement jusqu'à 31 \% (Rock et Schlaff, 1985).

Il est indiscutable que certaines synéchies en particulier fibreuses centrales ou isthmiques peuvent être des causes d'avortement à répétition (14 \% des 1973 patientes porteuses de telles synéchies (Rock et Zacur, 1983). Bien plus la levée de ces synéchies soit par les méthodes classiques, soit mieux sous contrôle hystéroscopique, permet le rétablissement d'un taux de grossesses normales dans $50 \%$ des cas. 
Mais la responsabilité des fibromes dans cette pathologie est diversement appréciée, suivant leur taille, leur nombre et surtout leur situation : la présence de myome sous muqueux est certainement un facteur d'échec et leur résection endoscopique une condition de succès. Mais il est aussi indispensable de tenir compte de l'âge des patientes, des troubles hormonaux contemporains, des lésions associées (adénomyose, hyperplasie endométriale, insuffisance lutéale). Si bien que le chiffre de $18 \%$ retenu comme fréquence dans leur intervention dans cette pathologie est certainement soumis à de nombreuses critiques. La myomectomie loin d'être systématique, mis à part les myomes sous muqueux, doit être précédée d'un bilan gynécologique systématique, sous peine d'ajouter à l'infertilité, une cicatrice utérine supplémentaire et inutile.

b) Les facteurs génétiques.

Si l'on décèle une anomalie chromosomique pour 175 nouveau-nés vivants, on sait, par contre, que $60 \%$ des avortements du $1^{\text {er }}$ trimestre (entre 3 et 7 semaines) sont dus à une anomalie chromosomique. Les travaux de Boué et Boué (1977) ont bien montré qu'il s'agissait surtout d'anomalies du nombre de chromosomes [monosomie $X(15$ à $20 \%)$, trisomie d'un autosome surtout $(16,50$ à $60 \%$ ), triploïdies (12 à $20 \%$ ), tétraploïdies (3 à $7 \%$ ) et mosaïques d'anomalies de structure (3 à $6 \%$ )]. Ces anomalies du nombre de chromosomes sont dues à une mauvaise ségrégation des chromosomes pendant la gamétogenèse masculine ou féminine, lors des première ou deuxième divisions méiotiques. On a généralement invoqué pour expliquer l'arrêt du développement foetal dans ces cas, un ralentissement de la croissance cellulaire et un asynchronisme dans les phénomènes d'induction, de maturation et de différenciation surtout des tissus embryonnaires. Mais deux remarques subsistent: comment expliquer qu'une même anomalie chromosomique comme la trisomie 21, puisse être la cause d'un avortement précoce ou d'une naissance d'un sujet viable mal formé ? D'autre part, il est regrettable que dans le cadre des avortements à répétition on n'ait pas recherché si la même anomalie chromosomique expliquait à chaque fois l'échec de la reproduction.

A défaut de cette preuve, plusieurs études ont fait le bilan des anomalies chromosomiques parentales dans le cadre des fausses couches à répétition. Une récente compilation, faite par Tharapel et al. (1985), sur 79 séries publiées, comportant 8208 femmes et 7834 hommes a révélé une prévalence d'anomalies chromosomiques majeures de 2,9\%. Ce chiffre est six fois plus élevé que celui d'une population de couples normaux féconds. Dans la grande majorité de ces études, il est noté une prédominance d'anomalie chez la femme, comme si chez l'homme une anomalie majeure conduisait plutôt à une stérilité. Mais aucune démonstration véritable ne peut expliquer ce fait. On relève que $50 \%$ de ces anomalies parentales sont des translocations réciproques équilibrées, $24 \%$ de translocations robertsonniens de chromosomes acrocentriques, $12 \%$ de mosaïques des chromosomes sexuels $X$, le reste étant constitué d'inversion péri ou paracentriques. La fréquence des translocations réciproques a été évaluée à $6 \%$ pour des couples présentant des avortements à répétition soit 10 fois plus que la moyenne générale. Cependant, il est assez étonnant de constater que la fréquence de ces anomalies n'est corrélée ni avec le nombre d'échecs de la reproduction, ni 
avec la présence d'enfants normaux ou non (Trochet-Royer et al., 1981). Enfin l'attitude concernant la demande de caryotype chez les parents après avortement reste nuancée : en effet pour certains (Trochet-Royer et al., 1981) s'il s'est agi précédemment d'une anomalie numérique (monosomie, trisomie ou triploides), accident fortuit de la méiose, il n'y avait pas lieu de faire une recherche chez les parents. Pour d'autres, par contre, (Tharapel et al., 1985), plus de deux avortements dans le même couple, justifie un examen systématique des caryotypes des parents et un caryotype de toutes les fausses couches à venir.

B) Parmi les causes probables, il faut ranger: les facteurs endocriniens, les causes infectieuses et toxiques et les troubles du métabolisme.

1) II n'y a aucune preuve tangible de l'influence d'une hypothyroïdie sur les avortements à répétition, si ce n'est des observations éparses de patientes ayant une fixation basse $\mathrm{d}^{\prime} \mathrm{l}^{131}$ ou de succès sans cas contrôle dus à l'administration quotidienne de thyroxine. Par contre le débat reste largement ouvert entre les tenants et les adversaires de l'insuffisance lutéale. Pour les premiers, cette incidence varie de $3,7 \%$ à $60 \%$ suivant les séries. Ces très grandes différences sont dues à l'appréciation même de l'insuffisance lutéale : faut-il l'apprécier avant toute grossesse (taux de progestérone inférieurs à $10 \mathrm{mg} / \mathrm{ml}$ pour deux dosages successifs dans la phase lutéale), sur un retard de 2 jours dans la datation de l'endomètre suivant les critères de Noyes (1950) ou sur une insuffisance de sécrétion de corps jaune dans les premières semaines de la gestation? Outre que ces constatations ne sont pas reproductibles d'un cycle à l'autre chez la même patiente, il existe souvent une non-concordance entre les sécrétions de progestérone et l'état de l'endomètre. Pour mieux illustrer ces contradictions, il est intéressant d'analyser un article récent de Daya et al. en février 1988 : après avoir fait une étude critique des différentes publications antérieures, les auteurs se proposent a) de prouver chez 65 femmes présentant des avortements à répétition, l'existence d'un taux de progestérone plasmatique $(P)$ inférieur à celui d'un groupe témoin b) de montrer qu'il existe une corrélation entre ces taux de $P$ et l'état endométrial c) de démontrer l'efficacité d'un traitement par la progestérone (50 mg par voie vaginale dès le $3^{\mathrm{e}}$ jour de la phase lutéale) estimée soit par la correction du taux de $\mathrm{P}$, soit par la survenue et la poursuite d'une grossesse. Une étude statistique très sérieuse doit venir étayer ces trois réponses. A la $1^{\text {re }}$ question, une réponse est apportée : le taux de $21 \mathrm{nmol} / \mathrm{l}$ est celui qui permet de discriminer les cycles normaux et les cycles à phase lutéale déficiente. C'est justement quand ce taux est inférieur à ce chiffre au $21^{\mathrm{e}}$ jour du cycle que le diagnostic serait établi avec $70 \%$ de sensibilité et $71 \%$ de spécificité. Mais un certain nombre de critiques méritent d'être faites: si l'intérêt de ce travail consistait dans l'appréciation des taux de $P$ jour après jour dans la phase lutéale, il est à noter que ces taux ne sont pas évalués jour après jour chez la même femme, en fait il s'agit de moyenne à un jour donné sans préciser l'évaluation de ces taux chez chaque patiente. De plus, la comparaison statistique entre les courbes normales et pathologiques en prenant la moyenne et l'écart standard de la moyenne, ne fait apparaître aucune différence. Les auteurs sont obligés d'avoir 
recours à une représentation logarithmique de taux de $P$ pour faire apparaître une différence et encore celle-ci n'est-elle spécifique et sensitive que pour $70 \%$ des cas.

II n'existe d'autre part aucune corrélation entre les sécrétions des stéroïdes et l'état de l'endomètre.

Enfin la justification thérapeutique est plus que douteuse : en effet si la $P$ plasmatique évolue dans les zones normales sur la courbe, l'efficacité n'est que partielle: sur les 43 femmes traitées, 24 furent enceintes et $17 \%$ d'entre elles eurent un avortement spontané. Il est bien évident que la démonstration pour être complète aurait dû nécessiter une étude en double aveugle placebo contre progestérone, et surtout une étude histologique préalable montrant la correction histologique au $21^{\mathrm{e}}$ jour.

Pour clore ce chapitre et bien qu'il ne s'agisse pas d'avortement à répétition nous nous devons de rappeler a) qu'aucune étude de supplémentation lutéale dans les fécondations in vitro n'a montré de supériorité par rapport aux séries non traitées ; b) que dans notre propre expérience sur 18 dons d'ovocytes, nous avons obtenu $30 \%$ de grossesses dont les $2 / 3$ évolutives avec des taux de $P$ aux alentours de $5 \mathrm{ng} / \mathrm{ml}$ et des biopsies d'endomètres très correctes ; c) que l'étude des récepteurs de l'endomètre non seulement en biochimie, mais aussi en immunocytochimie pourra nous donner des renseignements complémentaires intéressants. Enfin si aucune preuve sérieuse de l'efficacité thérapeutique de la $P$ n'a pu être apportée, il faut signaler à la décharge de ce traitement qu'aucun argument n'a pu lui attribuer la responsabilité de certaines malformations telles que la réduction de la taille des cotes, les anomalies vertébrales, anales, trachéo-oesophagiennes, ou la transposition des gros vaisseaux.

2) Si l'on considère qu'une sérologie positive ou que même l'isolement d'un germe à partir de l'endocol n'est pas une preuve suffisante pour expliquer les avortements spontanés et encore moins les avortements à répétition, on comprend pourquoi il faut être très prudent pour retenir cette étiologie. Une seule fois un cyto-mégalovirus a été isolé de l'endomètre, cela a été aussi très rarement le cas dans les suspicions de Listeria ou d'infections par chlamydiae trachomatis. Seuls Candiani et al. (1987) retrouvent une infection endométriale à chlamydiae chez $22 \%$ des patientes présentant des avortements à répétition contre $6 \%$ dans une population témoin. Le rôle du toxoplasme reste très discuté, en effet la preuve sérologique n'est pas convaincante, seule une culture de tissu avec inoculation chez le rat ou recherche en immunofluorescence pourrait être déterminante. Par contre des preuves de l'intervention du mycoplasme Tiny sont plus convaincantes, si l'on en juge par les travaux de Caspi et al. (1972), Stray-Pedersen et Lorentzen-Styr (1977). Ce rôle d'uréaplasma est d'autant plus vraisemblable pour certains auteurs que le traitement par des cyclines, a été fréquemment couronné de succès. Mais aucune étude prospective, avec témoins ne permet d'avoir une opinion définitive sur ce sujet. Par contre, la culpabilité de l'herpes virus telle qu'elle peut être rapportée par la culture du virus, est plus que vraisemblable (jusqu'à $35 \%$ d'avortements à répétition contre $10 \%$ dans une série contrôle).

3) Le rôle du diabète, longtemps considéré comme responsable de 15 à $30 \%$ d'avortement à répétition est par contre, maintenant très diversement apprécié. 
Une récente étude de Crane et al. (1981) ne retrouve pas cette incidence quand ils comparent un groupe de patientes ayant un diabète gestationnel, un diabète franc et un groupe témoin. II est vrai que dans ces cas, la correction thérapeutique correcte du diabète peut expliquer ces constatations.

4) Enfin parmi les causes actuellement en cours d'évaluation il faut retenir les causes immunologiques. Dans ce cadre, il convient de distinguer deux grands chapitres : celui de l'auto-immunité et celui des réactions allogéniques intéressant le couple. Parmi les maladies auto-immunes, il en est une qui sans conteste est source d'avortement à répétition : c'est le lupus érythémateux disséminé (5 à $40 \%$ de fausses couches à répétition suivant les auteurs). Cependant pour bien cerner le problème il convient de préciser un certain nombre de points.

a) Tout d'abord, la physiopathologie du syndrome:

- Une première cause pourrait être une atteinte trophoblastique par des anticorps lymphocytotoxiques ayant une réaction croisée avec les antigènes trophoblastiques.

- La deuxième cause serait l'existence d'un anticoagulant circulant de type antithromboplastine, décrit pour la première fois par Nilsson et al. en 1975, repris dans un syndrome particulier par Soulier et Boffa (1980) associant en plus et paradoxalement des accidents thrombo-emboliques, et enfin défini par Edelman et al. $(1986 a, b)$ comme le pivot d'une véritable maladie abortive auto-immune. Cet inhibiteur, une immuno-globuline acquise de la classe $\lg \mathrm{G}$ ou $\lg \mathrm{M}$ a une action anti-phospholipide dirigée contre la fraction phospholipidique de la prothrombinase, expliquant la survenue d'une thrombopénie par action sur les phospholipides plaquettaires ou une positivité dissociée de la réaction sérologique de la syphilis. De plus Carreras et al. (1981) ont montré que ces immuno-globulines, responsables de l'activité anti-coagulantes, diminuaient la libération des prostacyclines (PGI2) par les cellules endothéliales, qui sont des agents vasodilatateurs et anti-agrégant plaquettaire puissant. Ainsi pourrait-on expliquer les thromboses au sein de la circulation fœto-placentaire et les conséquences sur la grossesse.

- Enfin, la troisième explication, en particulier des morts in utero, pourrait être le passage d'IgG maternel transplacentaires, qui pourraient réagir avec le système de conduction cardiaque, provoquant une réaction inflammatoire, une fibrose et un bloc auriculo-ventriculaire (Reymond et al., 1987).

b) Sur le plan clinique, I'association entre lupus et syndrome abortif avec trouble de la coagulation est loin d'être absolue. On parle alors de formes prémonitoires de LED, ou de formes apparentées.

c) C'est pourquoi sur le plan biologique, il est nécessaire:

— de mettre en évidence cet anti-coagulant circulant sur plasma pauvre en plaquettes par essentiellement deux tests: le test de thromboplastine dilué du malade sur celui du témoin (le test est positif quand il égale ou dépasse 1,2) et 
le taux de céphaline activé qui marque l'existence d'un anti-coagulant circulant quand le temps du malade est supérieur de 10 à celui du témoin et que cet allongement n'est pas corrigé par le plasma témoin ;

- mais aussi de rechercher des anticorps témoins de la maladie auto-immune : antinucléaire $(>1 / 100)$, anti-mitochondrie ou thyroïdien $(>1 / 40)$, anti-DNA $(>1 / 25)$, anti-thyroglobulines $(>1 / 80)$ et de Waaler-Rose $(>1 / 32)$, les anticorps anti-nucléaires solubles (anti-RO), enfin les anticorps anti-cardiolipiniques par la technique ELISA.

III) Sur le plan diagnostique, une récente étude prospective sur 130 patientes ayant présenté des avortements à répétition, menée par Edelman et al. (1986a,b) avec notre participation, permet de tirer des conclusions intéressantes mais non définitives : ces patientes n'avaient aucun antécédent de maladie auto-immune, mais $10 \%$ d'entre elles avaient un anticorps anti-thromboplastine de typique lupique, et la moitié des femmes de ce groupe avaient des réactions biologiques de type auto-immunes. Deux points sont aussi à souligner : d'une part l'association statistiquement démontrée entre anti-thromboplastine et avortement à répétition, d'autre part l'ambiguïté existant entre le lupus et la maladie abortive peut être à expression uniquement gynéco-obstétricale.

IV) Enfin sur le plan thérapeutique, depuis les premiers résultats publiés par Lubbe et al. (1984) et par Brauch et al. (1985), Edelman et al. (1986a,b) ont utilisé la corticothérapie à doses modérées ( 20 à $40 \mathrm{mg} /$ jour) dès le début de la gestation ou dès la $12^{\mathrm{e}}$ semaine; elle a été poursuivie à doses dégressives, associée ou non à un anti-agrégant plaquettaire, avec sur de courtes séries préliminaires des résultats très encourageants (jusqu'à $80 \%$ de succès) mais il manque un vrai essai en double aveugle pour conclure véritablement.

Les réactions immunologiques, allogéniques intéressent le couple :- Pour tolérer, la greffe semi-allogénique représentée par le fœtus, le placenta doit établir une barrière et bloquer les précurseurs des lymphocytes $T$ cytotoxiques et la mère élaborer des anticorps bloquants. Or une histo-compatibilité entre père et mère ne favorise pas cette protection, particulièrement si elle intéresse le système HLA d'histo-compatibilité, porté par le chromosome 6 . Ainsi la réponse maternelle normale se traduit-elle par la fabrication d'anticorps anti HLA paternels exprimés sur les cellules fœtales. Lors de fausses couches à répétition (FCR), une absence d'anticorps HLA a été constatée chez la mère (Menge et Beer, 1985). De plus, normalement, le sérum maternel, supprime la réactivité cellulaire aux antigènes HLA paternels, mesurés par l'inhibition de la culture mixte lymphocytaire. Cette inhibition de la culture mixte lymphocytaire ne se produit plus en cas de FCR.

Mais en fait la question est bien plus complexe : d'une part, il a été suggéré (Mc Intyre, 1983) que des antigènes (TLX) ayant des réactions croisées avec des antigènes HLA, pouvaient être les inducteurs de cette réaction bloquante, d'autre part, ce complexe majeur d'histo-compatibilité est très diversifié : antigènes de classe I contrôlés par les locus A.B.C., antigènes de classe II sous le contrôle des locus DR/D, DO et DP. Mais les locus $A$ comprennent eux-mêmes 17 antigènes 
connus, 30 antigènes pour le locus $B, 5$ pour le locus $C, 14$ pour le locus DR, et 3 pour le locus DQ (Gebukrer et al., 1987). Ils sont portés par les lymphocytes et reconnus par des réactions sérologiques.

Pour étayer cette hypothèse il fallait :

$\left.1^{\circ}\right)$ Démontrer une différence d'homologie dans ce système entre les couples fertiles contrôles et les couples à fausses couches répétées sans explication habituelle. II n'y a pas sur ce point encore de consensus : certains ne l'ont pas observés (Barnes et al., 1984; Olesenberg Perstiz, 1983) certains l'admettent (Reznikoff-Etievant et al., 1984) ; d'autres sont plus nuancés et reconnaissent que cette homologie n'est pas très élevée (Gill, 1983). Récemment l'étude personnelle de Reznikoff (1987) associée à 20 autres études, sur 1154 couples présentant des FCR, comparés à 1071 couples normaux, montre une augmentation significative de l'homologie HLDDR mais également HLAB. Cependant, d'autres études soulignent la complexité du Locus DR et les difficultés d'affirmer I'homologie, même s'il existe une identité sérologique, qui ne correspond pas forcément à une identité allogénique. Bien plus, Christiansen et al. (1988), ont montré outre l'absence d'homologie dans le système HLA chez des couples ayant des FCR ; l'existence chez les sœurs de ces patientes de locus HLA identiques, avec échecs de la reproduction. Ils postulent qu'il existerait, chez l'homme lié au système HLA, un t-glc complex analogue à celui des rongeurs responsables de résorptions embryonnaires.

$2^{\circ}$ ) Prouver par le développement d'anticorps anti-paternels, le succès de la thérapeutique dite de "vaccination" par les lymphocytes paternels. De très nombreuses démonstrations ont été apportées par différentes études (Mowbray et al., 1985), essentiellement en France par Netter et Reznikoff (1987). Leurs résultats se rapprochent de ceux de Beer (1986), et de Mobray (1985). Ainsi sur une série de 31 femmes immunisées, ayant développé 16 grossesses, le taux de succès est de $93 \%$, alors que dans trois cas sans immunisation, il y eut trois fausses couches. Même, si la thérapeutique, de préférence avec les lymphocytes paternels, est bien codifiée [200 millions de leucocytes sont séparés par gradient de densité et injectés à raison de 150 millions en IV et 50 en IM et intra-dermique. Une à trois injections à 1 mois d'intervalle jusqu'à obtention d'une immunisation anti-paternelle vérifiée 3 semaines après chaque injection]; - même si des précautions sont prises pour les transfusions (notamment: recherche de l'antigène $\mathrm{Hbs}$, anti HIV, anti CMV), beaucoup d'auteurs ont une attitude restrictive : soit qu'ils redoutent certaines complications ultérieures chez la femme ou le fœetus (en rappelant que chez l'animal de laboratoire, on a noté la survenue de malformations, de leucémies ou de lymphomes), soit qu'ils regrettent l'absence de séries plus étoffées avec cas contrôles. Enfin, beaucoup de tenants de cette thérapeutique, n'excluent pas que le développement de ces anticorps ne soient que les témoins innocents de la réponse immune de tolérance, comme le rappelle Reznikoff (1987).

$3^{\circ}$ ) Enfin s'il n'a pas été démontré que des anticorps anti-sperme chez I'homme, ou une incompatibilité dans les groupes $A B O$ pouvaient être la cause d'avortements à répétition, par contre, une incompatibilité dans le système $P$ (Rock et al., 1985) peut être la cause de pertes embryonnaires répétées et une 
plasmaphorèse débarrassant la mère des anticorps anti $P$ pouvait être couronnée de succès. En fait, la stratégie thérapeutique ne peut dépendre que du bilan diagnostique. On a vu, compte tenu des différentes étiologies et de leur fréquence, les multiples composantes du bilan qu'il convient classiquement de dresser. Mais plutôt que de demander, comme cela est souvent le cas, une batterie d'examens complémentaires qui vont de l'hystérographie à l'inhibition de la culture mixte lymphocytaire, il convient d'ordonner cette demande et notamment de préciser par un simple interrogatoire si les avortements sont précoces avant 8 semaines, ou tardifs, s'ils sont primaires (sans aucune naissance préalable) ou secondaires, et enfin s'ils sont entrecoupés ou non de naissances vivantes ou de morts in utero. La notion de terrain, nous paraît essentielle en insistant notamment sur une auto-immunisation probable, une infertilité préalable, une participation hormonale. Nous ne passerons donc pas en revue, tous les examens qui pourraient alors être demandés, mais nous voulons insister sur les tendances actuelles en matière d'échecs de l'implantation.

Ainsi Brambati et al. (1988) montrent dans leur série que dans les 200 cas d'avortements constatés entre 6 et 8 semaines, le taux d'anomalie chromosomique est de $85 \%$ et de $77 \%$ dans les 56 cas de 9 à 11 semaines, contre $5,7 \%$ dans 1259 cas de grossesses évolutives.

Les mêmes auteurs insistent sur l'utilité de l'échographie vaginale utilisant une sonde de $7,5 \mathrm{MgHz}$; elle permet dès 6 semaines de grossesse de donner des appréciations non seulement sur les battements cardiaques, l'état du sac, mais aussi l'évolution de la croissance cranio-caudale ainsi que sur des détails comme la vésicule ombilicale, le coelome extra-embryonnaire et le cordon. Ainsi dans les cas d'une distance cranio-caudale inférieure ou égale au $5^{\mathrm{e}}$ pecentile avant la $10^{\mathrm{e}}$ semaine, non seulement une mort fœtale survient dans $30 \%$ des cas, mais une aneuploïdie est alors rencontrée dans $80 \%$ de ces cas. De plus l'absence de visualisation de la vésicule ombilicale, ou son non-développement entre la $6^{\mathrm{e}}$ et la $8^{\mathrm{e}}$ semaine est un signe très péjoratif en faveur d'une aneuploïdie ou d'une malformation. Ceci est en tout cas incontestablement vérifié dans les expériences sur le rat.

Schats et al. (1988), ont précisé l'existence d'un asynchronisme dans l'implantation de grossesses multiples après fécondation in vitro. Cette démonstration a été faite en ultrasonographie, à partir du $25^{\mathrm{e}}$ jour après l'ovulation : ainsi sont apparues des différences dans le volume des sacs, la longueur craniocaudale des embryons et surtout l'apparition des bruits du cœur ( 2 à 3 jours parfois). Cet asynchronisme pourrait dans certains cas expliquer certains retards d'implantation avec disparition secondaire des sacs.

D'autres auteurs se sont attachés à montrer l'importance de différents marqueurs comme l'alpha fœto-protéine dont la diminution serait associée à certaines aneuploïdies comme la trisomie 21. Cette diminution serait en rapport avec un mauvais fonctionnement de la vésicule ombilicale ou plus tard du foie fœtal.

Certaines glycoprotéines comme le PAPP-A d'origine trophoblastique, pour d'autre d'origine endométriale, la PP12 sécrétée par les cellules déciduales 
seraient aussi de bons marqueurs de la vitalité fœtale, peut être même plus sensible que la sécrétion de hCG (Howell et al., 1988).

D'autres (Beier-Hellwig et al., 1988) poussent plus loin l'exploration dans ce sens en s'adressant à l'électrophorèse des protéines endométriales étudiée durant la phase lutéale pour tester sa réceptivité, ou en dosant in vitro la sécrétion par les lymphocytes maternels d'une substance hCG-like en cas de grossesse, ou même en dosant dans le liquide amniotique deux nouveaux antigènes fotaux FA1 et FA2 dont le dernier serait plus spécifiquement en relation avec les composantes du tissu conjonctif.

Toutes ces tentatives prouvent que la « saga » des avortements à répétition est loin d'être terminée. Deux remarques s'imposent cependant: jusque-là le responsable semblait devoir être la mère, de nombreux arguments militent maintenant en faveur d'une interaction parentale; bien plus, le produit de conception apparaît de plus en plus comme l'objet de menaces dès son avènement, et les explorations mises à la disposition du clinicien permettent actuellement sinon de toujours éviter ces accidents, du moins de mieux les comprendre.

$27^{e}$ Réunion de la Société française pour l'Etude de la Fertilité. Paris, 29, 30 sept., $1^{\text {er }}$ oct. 1988.

\section{Références}

AUDEBERT A. J. M., CITTADINI E., COGNAT M., 1983. Avortements à répétition et malformations utérines. Contracept. Fertil. Stéril., 11, 1109-1116.

BARNES J., HART C. A., FRANCIS W. J., 1984. Determinants of immunological responsiveness in recurrent spontaneous abortion. Transplantation, 38, 280-284.

BEER A., 1986. New horizons in the diagnosis, Evaluation and therapy of recurrent spontaneous abortion. Clinics Obst., Gynaecol., 13, 115-124.

BEIER-HELLWIG K., STERZIL K., BOUN B., BEIER H. M., 1988. Critical evaluation of endometrial receptivity by uterine secretion electrophoresis in IVF-therapy. Abstract $N^{\circ} 1039$. 4th Annu. Meet. ESHRE, Barcelone.

BOUÉ A., BOUÉ J., 1977. Le rôle des anomalies chromosomiques dans les échecs de la reproduction. J. Gyn. Obst. Biol. Repr., 6, 5-21.

BRAMBATI B., LANZANI A., 1987. A clinical look at early post-implantation pregnancy failure. Human Reprod., 2, $401-405$.

BRAUCH D. W., SCOTT J. R., KOCHENOUR N. K., HERSHGOLD E., 1985. Obstetric complications associated with the lupus anti-coagulant. N. Engl. J. Med., 313, 1322-1326.

CANDIANI G. B., FEDELE L., 1987. Recurrent spontaneous abortion : stage of the art and new horizon. Acta Eur. Fertil., 18, $91-104$.

CARRERAS L. O., DEFREYN G., MACHIM S. J., VERMYLEN J., DEMAN R., SPITZ B., VAN ASSCHE A., 1981. Arterial thrombosis intra-uterine death and lupus anti-coagulant: detection of immunoglobulin interfering with prostacyclin formation. Lancet, i, 244-246.

CASPI E., SALAMON F., SOMPOLINSKY D., 1972. Early abortion and mycoplasme infection. /sr. J. Med. Sci., 8, 122-127

CHRISTIANSEN O. B., LAURITZEN J. G., RIISOM M. K., GRUNNET N., 1988. A human analog to the T/GRC-complex in rodents seems to cause a part of idiopathic habitual abortions. Abstr. $n^{\circ}$ 248. 4th Annu. Meet. ESHR, Barcelone.

CRANE J. P., WAHL N., 1981. The role of material diabetes in repetitive spontaneous abortion. Fertil. Steril, 36, 477-486. 
DAYA S., WARD S., BURROWS E., 1988. Progesterone profiles in luteal phase defect cycles and outcome of progesterone treatment in patients with recurrent spontaneous abortion. $A \mathrm{~m} . \mathrm{J}$. Obstet. Gynecol., 158, 225-232.

EDELMAN Ph., ROUQUETTE A. M., VERDY E., ELIAS A., CABANE J., CORNET D., BARRAT J., CHAVINIE J., SALAT-BAROUX J., SUREAU C., 1986a. Autoimmunity, fetal losses, lupus anticoagulant : beginning of systemic lupus erythematosus or new autoimmune entity with gynaecol-obstetrical expression. Human. Reprod., 1, 295-297.

EDELMAN Ph., VERDY E., ROUQUETTE A. M., CORNET D., COMBRISSON A., BARRAT J., CHAVINIE J., SALAT-BAROUX J., SUREAU C., 1986b. Désordres immunologiques de la coagulation dans la malade abortive. Presse. méd., 15, 961-964.

FRENCH F. E., BIERMAN J. E., 1962. Probabilities of fetal mortality. Public Health Reports, 77. $N^{\circ} 10$.

GEBUKRER L., MELLIER G., DOILLON H., THOULON J. M., BETNEL H., 1987. Absence d'homologie HL-A dans les couples présentant des fausses couches à répétition. $R e v$. Fr. Gyneco-Obstet., 82, 699-703.

GILL T. J., 1983. Immunogenetics of spontaneous abortions in humans. Transp/antation, 35, 1-6.

HERTIG A., ROCK J., 1959." A series of potentially abortive ova recovered from fertile women prior to the first missed menstrual period. Amer. J. Obstet. Gynec., 58, 968.

HOWELL R. J. S., OLAJIDE F., GRUDZINKAS J. O., CHAND T., 1988. Circulating levels of placenta protein 12 (PP12) and hCG following RU 38486 and gemeprost for termination of first trimester pregnancy. Abstr. $\mathrm{N}^{\circ} 59$, 4th Annu. Meet. ESHRE, Barcelone.

LECOUTOUR X., BOURGEOT P., SEGARD G., VERBRACHEL L., LEROY J. L., PUECH F., DELECOUR M., 1986. L'avenir obstétrical des utérus mal formés. Rev. Fr. Gynecol. Obst., 81, 357-362.

LERIDON H., 1973. Démographie des échecs de la reproduction. In Aspects biométriques de la fécondité humaine. Presses Universitaires de France, Paris.

LERIDON H., 1977. Human fertility. The University of Chicago Press. Chicago.

LUBBE W. F., BUTLER W. S., PALMER S. J., LIGGINS C. G., 1984. Lupus anticoagulant in pregnancy. Br. J. Obstet. Gyneco., 91, 357-363.

McINTYRE J. A., 1983. Human trophoblast-lymphocyte cross-reactive (TLX) antigens define a new alloantigen system. Science, 22, 1135-1136.

MENGE A., BEER A., 1985. The significance of human leukocyte antigen profiles in human infertility, recurrent abortion and pregnancy disorders. Fertil. Steril., 42, 693-695.

MOWBRAY J. F., LIDDELL H., UNDERWOOD S. L., GIBBRINGS G., REGINALD P. W., BEARD R. W., 1985. Controlled trial of treatment of recurrent spontaneous abortion by immunisation with paternal cells. Lancet, i, $941-943$.

NETTER A., REZNIKOFF-ETIEVANT M. F., 1987. Fausses couches à réplétions idiopathiques. Rôle de l'immunité. Thérapeutique, 15, 547-549.

NILSSON I. M., AOTEDT B., HEDNER W., BEREZIN D., 1975 . Intra-uterine death and circulating anticoagulant (antithromboplasm). Acta Med. Scand., 197, 153-159.

NOYES R. W., HERTIG A., ROCK J., 1950. Dating the endometrial biopsy. Fertil. Steril., 1, 3-13.

OLESENBERG PERSTIZ E., AMAR A., SHENKEL J., SEGAL S., NELKEN D., BRANTBAR C., 1983. Mixed lymphocyte reactivity non responsiveness in couples with spontaneous abortions. Fertil. Steril., 39, 525-529.

REYMOND O., STADLER J. B., STALDER M., NGUYEN THE H., BOSSARD H., 1987. Lupus érythémateux, anticoagulant circulant et complications obstétricales. J. Gynecol. Obst. Biol. Reprod., 16, 207-211.

REZNIKOFF-ETIEVANT M. F., EDELMAN Ph., MULLER J. Y., PINON P., SUREAU C., $1984 . \quad$ HLA-DR locus and maternal fetal relation. Tissue Antigens, 24, 30-34.

REZNIKOFF-ETIEVANT M. F., 1987. Les fausses couches spontanées répétées. Rôle de l'immunité anti-paternelle et immunothérapie. Gynécologie, 38, 337-341.

ROCK J., ROSETTA S., HAYDEN O., BRAINE-NESS P., KICKLEN Th., NIEBYL J., $1985 . \quad$ Plasmapheresis for the treatment of repeated early pregnancy vastage associated with anti-P. Obstet. Gynecol., 66, 579-600.

ROCK J. A., ZACUR H. A., 1983. The clinical management of repeated early pregnancy vastage. Fertil. Steril., 39, 123-140. 
ROCK J. A., SCHLAFF W., 1985. The obstetric consequences of utero vaginal anomalies. Fertil. Steril, 43, 681-692.

RUDIGOZ R. C., DARGENT D., AUDRAN Ph., 1985. Le pronostic obstetrical des malformations utérines. Evolution des idées. Gynécologie, 36, 123-126.

SCHATS R., BRANDOMA G., CLEVERINGA L. M., LANKHORST I. S., JANSEN V., et C., 1988. Evidence of asynchronous implantation in IVF plural pregnancies. 4th Annu. Meet. ESHRE, Barcelone.

SOULIER J. P., BOFFA C., 1980. Avortements à répétition, thromboses et ACC-antithromboplastine. Presse méd., 9, 859-864.

STRAY-PEDERSEN B., LORENTZEN-STYR A. M., 1977. Uterine toxoplasma infections and repeated abortions. Am. J. Obstet. Gynecol., 128, 716.

THARAPEL A., SUGHANDI A., THARAPEL-BANNERMAN R., 1985 . Recurrent pregnancy losses and parental chromosome abnormalities : A review J. Obstet. Gynecol., 92, 899-914.

TROCHET-ROYER C., SALE B., JALBERT P., PISON H., RACINET C., BERNARD P., 1981. Avortements spontanés et anomalies chromosomiques parentales. Rev. Fr. Gynecol. Obstet., 76, 196-199. 